\title{
1 Emergence and molecular basis of azithromycin resistance in typhoidal Salmonella in
}

\section{Dhaka, Bangladesh}

3 Yogesh Hooda ${ }^{1,2, \#}$, Senjuti Saha ${ }^{1,3, \#, *}$, Mohammad S. I. Sajib ${ }^{1}$, Hafizur Rahman ${ }^{1}$, Stephen P.

4 Luby $^{3}$, Joseph Bondy-Denomy ${ }^{4,5}$, Mathuram Santosham ${ }^{6}$, Jason R. Andrews ${ }^{3}$, Samir K. Saha ${ }^{1,7, *}$

$5{ }^{1}$ Child Health Research Foundation, Department of Microbiology, Dhaka Shishu Hospital,

6 Dhaka, Bangladesh

$7 \quad{ }^{2}$ MRC Laboratory of Molecular Biology, Cambridge, United Kingdom

$8 \quad{ }^{3}$ Division of Infectious Diseases and Geographic Medicine, Stanford University School of

9 Medicine, Stanford, CA, United States of America

$10{ }^{4}$ Department of Microbiology and Immunology, University of California, San Francisco, San

11 Francisco, CA, USA

$12{ }^{5}$ Quantitative Biosciences Institute, University of California, San Francisco, San Francisco, CA,

13 USA

$14{ }^{6}$ Department of International Health, Johns Hopkins Bloomberg School of Public Health,

15 Baltimore, MD, USA

$16{ }^{7}$ Bangladesh Institute of Child Health, Dhaka Shishu Hospital, Dhaka, Bangladesh

17

$18 \quad$ \#These authors contributed equally to the study

19

20 *Corresponding authors and lead contacts; send correspondences to Senjuti Saha

21 (senjutisaha@chrfbd.org) and Samir K Saha (samir@chrfbd.org) 


\section{Abstract}

24 With rising fluoroquinolone and ceftriaxone-resistant Salmonella Typhi, azithromycin, a

25 macrolide, has become the last oral drug available against typhoid. Between 2009-2016, we

26 isolated 1,082 Salmonella Typhi and Paratyphi A strains in Bangladesh, 13 (12 Typhi and 1

27 Paratyphi A) of which were azithromycin-resistant. When compared to 462 previously

28 sequenced Typhi strains, the genomes of the 12 azithromycin-resistant Typhi strains (4.3.1 sub-

29 clade, H58) harbored an exclusive non-synonymous single-point mutation R717Q in AcrB, an

30 RND-efflux pump. Expression of AcrB-R717Q in E. coli and Typhi strains increased its

31 minimum inhibitory concentration (MIC) for azithromycin by 11- and 3-fold respectively. The

32 azithromycin-resistant Paratyphi A strain also contained a mutation at R717 (R717L), whose

33 introduction in E. coli and Paratyphi A strains increased MIC by 7- and 3-fold respectively,

34 confirming the role of R717 mutations in conferring azithromycin resistance. With increasing

35 azithromycin use, strains with R717 mutations may spread leading to treatment failures, making

36 antibiotic stewardship and vaccine introduction imperative. 
Introduction

39 Typhoid and paratyphoid, collectively known as enteric fever, are among the most common

40 bacterial causes of morbidity worldwide, with the greatest burden in low- and middle-income

41 countries (GBD 2017 Typhoid and Paratyphoid Collaborators, 2019). Salmonella

42 enterica subspecies enterica serovars Typhi (Salmonella Typhi) and Paratyphi (A, B and C),

43 etiologies of enteric fever, cause an estimated 14 million illnesses and 136,000 deaths annually.

45 In the pre-antibiotic era, enteric fever mortality rates exceeded $20 \%$ in many areas, but

46 ampicillin, chloramphenicol and co-trimoxazole were instrumental in reducing the rates to $<1 \%$.

47 Resistance to all three antibiotics (referred to as multidrug resistance, MDR) emerged in late

48 1980's (Mirza et al., 1996), predominantly due to the rise and subsequent continental migration

49 of H58 haplotype (now referred to as 4.3.1), which contained the resistance genes either on

50 IncH1 plasmids or integrated within the chromosome (Holt et al., 2011; Wong et al., 2015,

51 2016). Fluoroquinolones soon became the most-commonly prescribed antibiotic (White et al.,

52 1996), but since the 2000's there have been increasing reports of decreased fluoroquinolone

53 susceptibility due to the acquisition of chromosomal mutations in the DNA gyrase and

54 topoisomerase IV genes (Roumagnac et al., 2006; Chau et al., 2007, Dimitrov et al., 2007; Pham

55 Thanh et al., 2016). In Bangladesh, $>99 \%$ of all Typhi and Paratyphi strains exhibit decreased

56 susceptibility to ciprofloxacin (Saha et al., 2018b). In 2011, WHO recommended ceftriaxone or

57 azithromycin for treating Salmonella Typhi non-susceptible to fluroquinolones (Balasegaram et

58 al., 2012). 
60 There have been sporadic reports of ceftriaxone-resistant Salmonella Typhi strains, (Saha et al.,

61 1999; Djeghout et al., 2018), but in 2016, an outbreak of extensively drug-resistant

62 (XDR) Salmonella Typhi, resistant to chloramphenicol, ampicillin, cotrimoxazole,

63 fluoroquinolones, and third-generation cephalosporins was recognized in Pakistan and to date

$64>1000$ cases have been confirmed (Andrews et al., 2018). Cephalosporin resistance of the XDR

65 strains was caused by the acquisition of a broad-spectrum beta-lactamase (bla-CTX-M-15) on an

66 IncY plasmid found in other enteric species. Typhoid patients in Pakistan are primarily being

67 treated with the last available oral option, the macrolide azithromycin, resistance to which is rare

68 (Klemm et al., 2018, Parry et al., 2015). This increasing use of azithromycin places selective

69 pressure for the emergence and spread of azithromycin-resistant isolates, raising concerns of

70 untreatable infections and increased mortality rates. Little is known about azithromycin

71 resistance in typhoidal Salmonella; while there are some sporadic reports on azithromycin

72 treatment failures (Molloy et al., 2010; Sjölund-Karlsson et al., 2011; Wong et al., 2015; Patel et

73 al., 2017), there are no data on the molecular mechanism of resistance.

75 In Bangladesh, Salmonella Typhi and Paratyphi A are the most common causes of bloodstream

76 infections in children $>2$ months of age and comprise of two-third of blood-culture positive

77 isolates in microbiology laboratories (Saha et al., 2017). Leveraging our surveillance system in

78 place for enteric fever, here we describe the emergence of azithromycin resistance among

79 typhoidal Salmonella in Bangladesh and identify the molecular basis behind this resistance.

\section{$81 \quad$ Result and Discussion}

82 Emergence of azithromycin-resistant Salmonella Typhi and Paratyphi A 
83 Between 2009 and 2016, through our enteric fever surveillance (Saha et al., 2017) in the

84 inpatient departments of the two largest pediatric hospitals of Bangladesh, we isolated 939

85 Salmonella Typhi and 143 Paratyphi A strains. Twelve of the Typhi and one of the Paratyphi A

86 strains were resistant to azithromycin, with disc diameters of $\leq 12 \mathrm{~mm}$, and minimum inhibitory

87 concentration (MIC) of $\geq 32 \mu \mathrm{g} / \mathrm{ml}$ (Parry et al., 2015). All 12 azithromycin-resistant Salmonella

88 Typhi strains were also MDR and were increasingly isolated since 2013 (Fig 1A), marking

89 gradual emergence of azithromycin-resistant Salmonella Typhi in Bangladesh. All patients lived

90 in Dhaka city, known to be endemic for typhoid (Figure S1).

91

92 Azithromycin resistant Salmonella Typhi harbors a mutation in the AcrB efflux pump

93 We sequenced the 12 azithromycin-resistant Typhi strains and found that all azithromycin-

94 resistant strains belonged to genotype 4.3.1 (H58), the most common genotype found in South

95 Asia (Tanmoy et al., 2018; Wong et al., 2015). In a whole-genome single nucleotide

96 polymorphism (SNP) tree, the 12 strains clustered together indicating that they are genetically

97 similar to one another and potentially arose for a single common parental strain (Figure 1B). To

98 identify the genetic basis of azithromycin resistance, we used three bioinformatic tools: SRST2

99 (Inouye et al., 2014), Resfinder (Zankari et al., 2012) and CARD (Jia et al., 2017) and to evaluate

100 the results obtained from these tools, we tested antimicrobial susceptibility against a panel of

101 nine other antibiotics (Figure 1C). While the tools successfully predicted the observed

102 susceptibility patterns for the nine antibiotics, no known azithromycin resistance mechanism was

103 identified (Figure 1C). Using PlasmidFinder (Carattoli et al., 2014) we identified two plasmids

104 found in these Salmonella Typhi strains: (i) IncQ1 (12/12 strains), containing genes for

105 ampicillin, co-trimoxazole and chloramphenicol resistance, and (ii) InFIB (12/12 strains), a 
106 plasmid commonly found in Salmonella Typhi strains (Kidgell et al., 2002; Park et al., 2018).

107 Both these plasmids were also present in closely related azithromycin-sensitive strains. The lack

108 of known azithromycin-resistance genes indicated a novel mechanism of azithromycin resistance

109 in these strains.

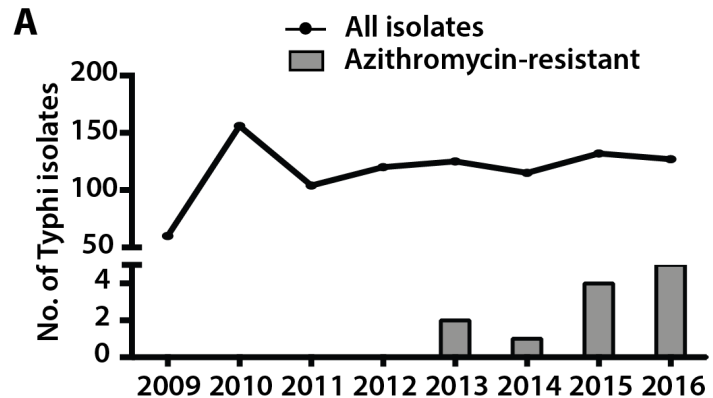

C

B

112 Figure 1: Emergence of azithromycin-resistant strains of Salmonella Typhi in Bangladesh

113 and their genomic analysis. A) Temporal distribution of 939 Salmonella Typhi isolates

114 included in the study. The number of isolates is shown as the line plot from 2009-2016. The

115 numbers of azithromycin-resistant strains isolated each year is shown in the bar plot.

116 Azithromycin-resistant strains were first isolated in 2013. B) Whole-genome SNP tree of 474

117 Salmonella Typhi strains isolated in Bangladesh previously (Tanmoy et al., 2018). The tree

118 highlights the different genotypes that are found in Bangladesh including the most prevalent

119 genotype 4.3.1 (H58 haplotype). The 12 azithromycin-resistant strains (colored in red) clustered 
120 together within the genotype 4.3.1. Salmonella typhimurium strain LT2 was used as an outgroup.

121 C) Predicted and experimentally determined antimicrobial susceptibility pattern of azithromycin-

122 resistant Salmonella Typhi strains and the most-closely related five azithromycin-sensitive

123 strains. The antimicrobial susceptibility was experimentally determined through disc diffusion

124 assay against a panel of 10 antibiotics. The predicted transmissible elements and antimicrobial

125 resistance markers are also shown.

126

127 We compared the sequences of these 12 azithromycin-resistant strains to that of 462 Typhi

128 strains that we had previously sequenced and genetically characterized (Tanmoy et al., 2018). In

129 the WGS SNP tree, we identified four unique SNPs, present only in the 12 azithromycin-resistant

130 strains, three of which were non-synonymous: STY2741 (codes for purN, a glycinamidine

131 ribonucleotide transformyltransferase), STY1399 (codes for a hypothetical protein) and

132 STY0519 (codes for AcrB, an inner membrane permease) (Figure 2A, Figure S2). For the first

133 two candidates, there is no evidence of their involvement in mediating antimicrobial resistance in

134 the literature. However, the third gene, $\operatorname{acr} B$ is part of a trans-envelope resistance-nodulation-

135 division (RND) efflux pump that has been previously reported to transport macrolides including

136 azithromycin across the bacterial cell envelope, making it the most promising candidate

137 (Nakashima et al., 2011). Mutations affecting expression of AcrB have been implicated in

138 macrolide resistance in Neisseria gonorrhoeae (Wadsworth et al., 2018). Furthermore, laboratory

139 mutagenesis studies in Escherichia coli have shown that mutations in $a c r B$ can lead to higher

140 macrolide efflux thereby contributing to resistance (Ababou and Koronakis, 2016). The SNP

141 observed in the 12 azithromycin-resistant Salmonella Typhi strains changed the arginine residue

142 (R) at position 717 to a glutamine (Q) (Figure 2B). R717 is a conserved residue on the

143 periplasmic cleft that acts as the entry portal for most drugs in AcrB (Figure 2C). In a previous

144 mutagenesis study, substitution of the arginine residue with an alanine (R717A) was found to

145 partially increase efflux of the macrolide erythromycin in E. coli (Yu et al., 2005). 
A
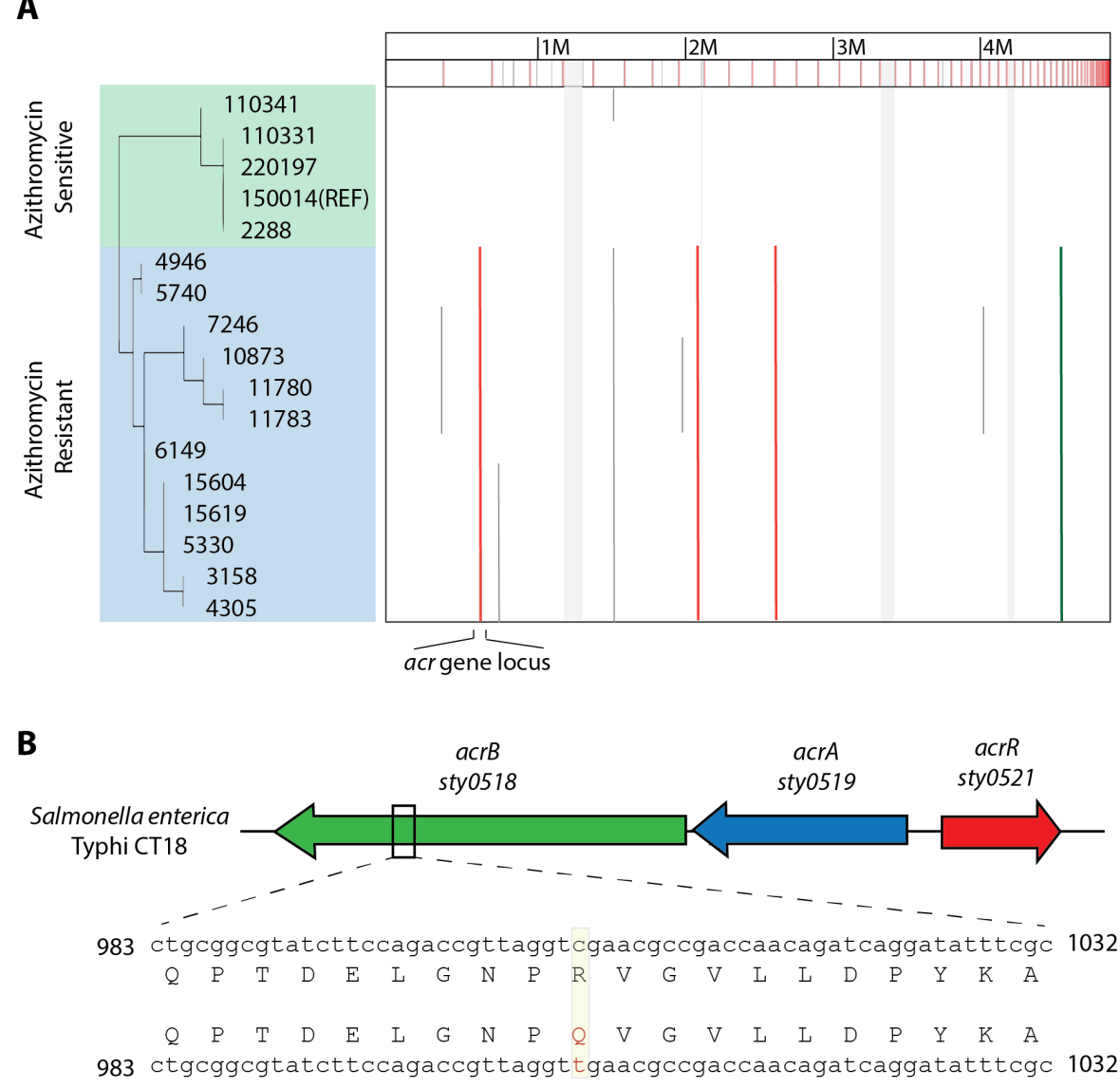

C

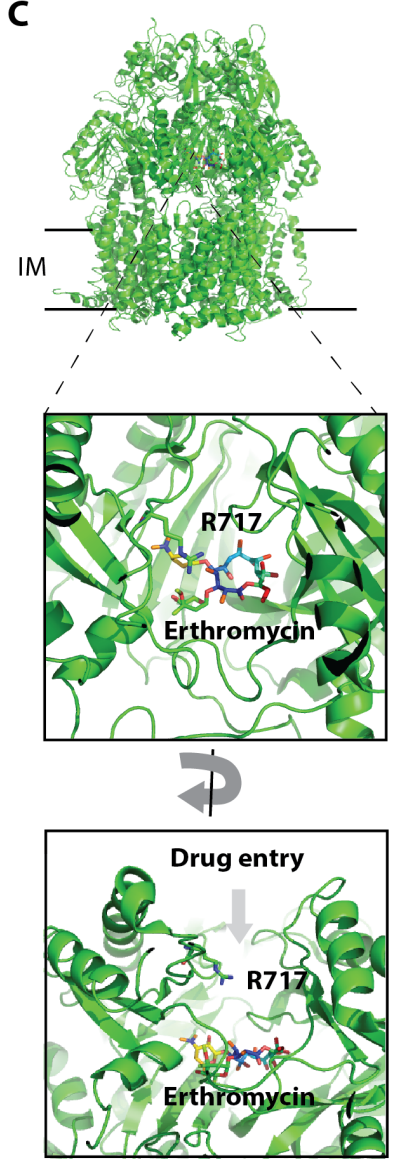

Figure 2: Identification of R717Q mutation on AcrB efflux pump as a cause of azithromycin resistance in Salmonella Typhi. A) Whole genome sequence alignment of 12 azithromycin-resistant and 5 genetically related azithromycin-sensitive Salmonella Typhi strains. Whole genome SNP detection and alignment was done using ParSNP and results were visualized in GinGR (Treangen et al., 2014). The single nucleotide polymorphisms (SNPs) unique to the resistant strains are highlighted with vertical lines. Four SNPs were identified: 3 nonsynonymous (shown as a red line) and 1 synonymous SNPs (shown as a brown line) that are exclusive to the azithromycin resistant strains. B) The acr gene cluster in Salmonella Typhi reference strain CT18. One of the SNPs found exclusively in azithromycin-resistant strains was mapped to the gene cluster composed of: $\operatorname{acr} A$ (STY0519) and $\operatorname{acr} B$ (STY0518), that encodes a periplasmic and inner membrane protein component of the RND-efflux pumps respectively, and $\operatorname{acrR}$ (STY0521), a transcriptional regulator of AcrA/B protein synthesis. The SNP was present on the $a \operatorname{cr} B$ gene and resulted in the change of an arginine (R) at position 717 to a glutamine (Q) residue on the encoded AcrB protein. C) $\mathrm{R} 717 \mathrm{Q}$ mutation is present at the periplasmic cleft of the proximal binding pocket on AcrB. Structure of E. coli AcrB (PDB ID: 3AOC) is shown in green with the macrolide erythromycin bound in the proximal drug binding pocket. AcrB is 
163 present in the inner membrane of the bacterial cells and drug molecules, including macrolides,

164 enter the AcrB pump through a periplasmic opening that leads to a proximal binding pocket. The

165 drug molecules are shuttled outside the cells through the proximal binding pocket with the help

166 of the proton motive force. R717 lines the entry the periplasmic opening.

\section{R717 mutations in AcrB confer azithromycin resistance}

169 We cloned $a c r B$ from azithromycin susceptible and resistant Salmonella Typhi strains into an $E$.

170 coli plasmid and introduced them into an E. coli strain that lacks the endogenous acrB (E. coli

$171 \triangle a c r B)$. Compared to $E$. coli strains containing empty plasmid or wild type $\operatorname{acr} B$, the strain

172 expressing AcrB-R717Q showed a smaller zone of disc clearance for both azithromycin (26.3

$173 \mathrm{~mm}$ vs $16.7 \mathrm{~mm}, p=0.0013)$ and erythromycin discs $(22.3 \mathrm{~mm}$ vs $11.7 \mathrm{~mm}, p$ value $=0.0009)$

174 and exhibited a 11-fold increase in azithromycin MIC (0.22 $\mu \mathrm{g} / \mathrm{ml}$ vs $2.7 \mu \mathrm{g} / \mathrm{ml}, p=0.0002)$

175 (Figure 3A, B). Certain AcrB mutations have previously been shown to effect transport of other

176 antibiotics such as ciprofloxacin (Blair et al., 2015), but the R717Q mutation did not change the

177 susceptibility patterns for any other nine antibiotics we tested (Figure S3). For further

178 confirmation of the effects of this mutation in Salmonella Typhi, we introduced the plasmids in

179 an azithromycin-sensitive Typhi strain and observed a 3-fold increase in MIC (5 $\mu \mathrm{g} / \mathrm{ml}$ vs 13

$180 \mu \mathrm{g} / \mathrm{ml}, p<0.0001$ ) in the presence of AcrB-R717Q (Figure 3C). The difference here is lower

181 compared to that seen in E. coli $\triangle a c r B$ plausibly because the Typhi strain contains endogenous

182 wild-type AcrB competing against the exogenous AcrB-R717Q that we artificially introduced.

183 Taken together, these results confirm that AcrB-R717Q leads to increased macrolide resistance. 


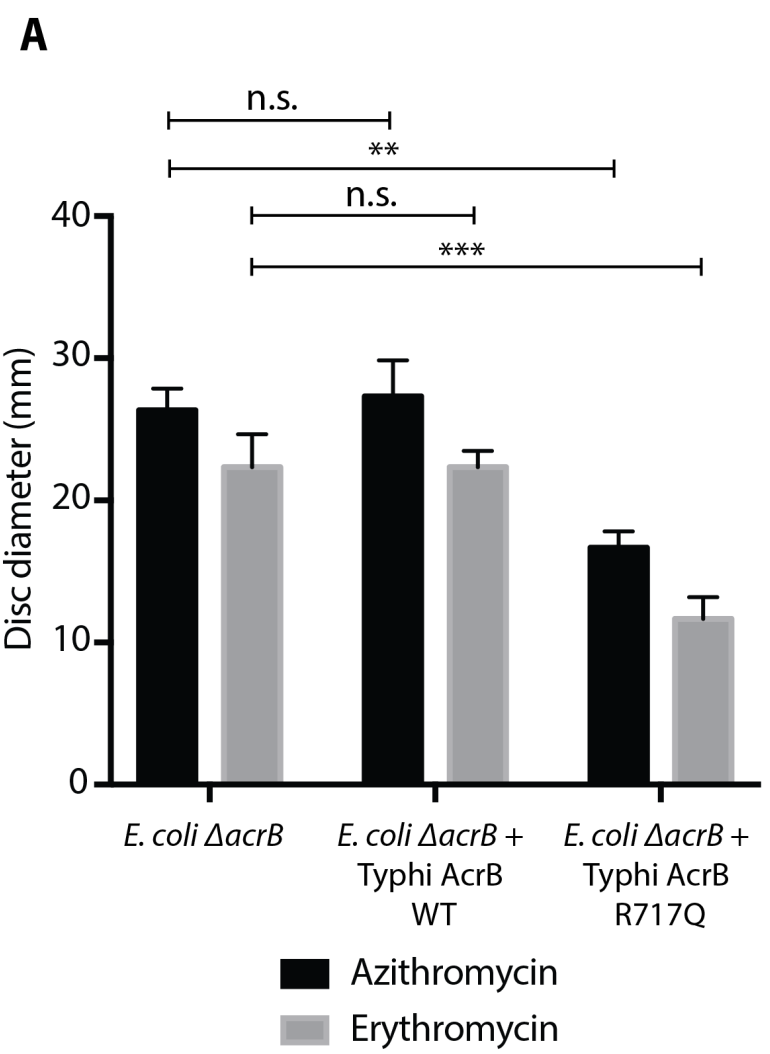

184 0.0001
B

C
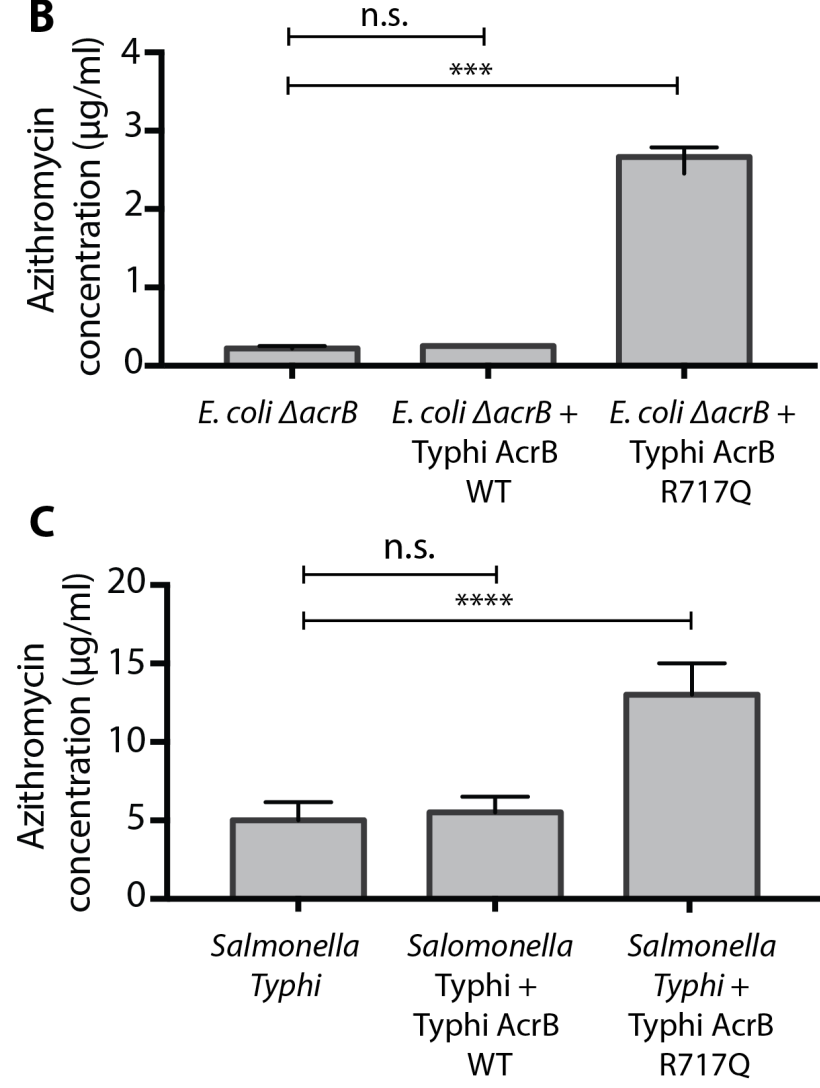

Figure 3: AcrB R717Q increases efflux of macrolides in E. coli and Salmonella Typhi strains. Quantification of results obtained from three biological replicates for (A) disc diffusion and (B) E-strip assays are shown for E. coli BW25113 $\triangle a c r B$ transformed with different plasmids. (C) Quantification of results obtained from at least three biological replicates from azithromycin E-strip assay in Typhi strain 4119. One way-ANOVA with multiple comparisons was used to test statistical significance. ns: $\mathrm{p}>0.05 ; * *: \mathrm{p} \leq 0.01 ; * * *: \mathrm{p} \leq 0.001, * * * *: \mathrm{p} \leq$

We conducted an extensive BLAST search to identify other typhoidal Salmonella strains with mutations in AcrB the NCBI database and found only one Salmonella Typhi strain isolated in Oceania in 2008 that contained the same R717Q mutation, however no AMR data were available for this strain (Wong et al., 2015). Interestingly, whole genome sequencing of the one azithromycin-resistant Salmonella Paratyphi A strain identified during our surveillance showed that this strain also contained a mutation in $a c r B$ which changed R717 to a leucine (L) (Figure 


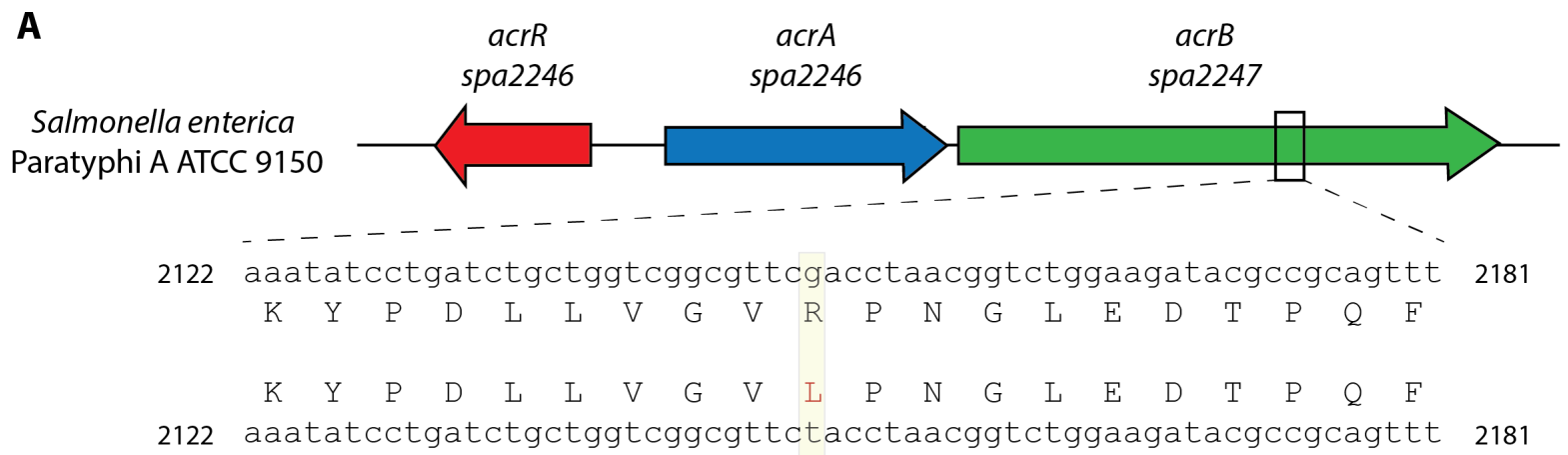

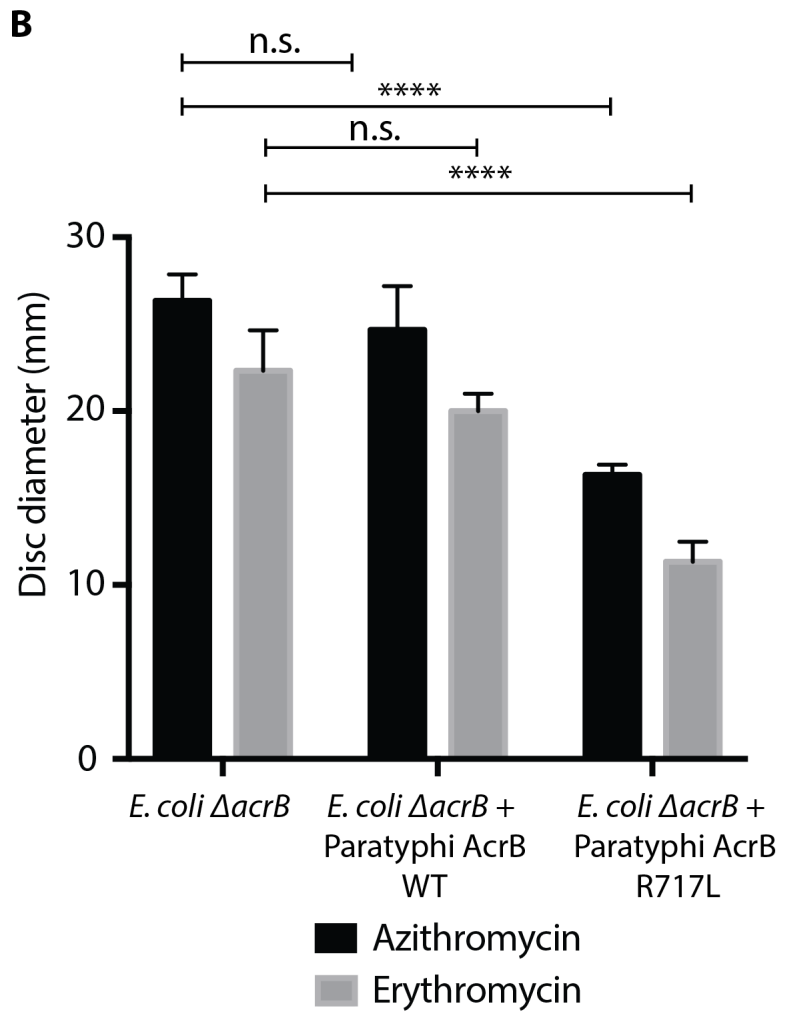

200

201

202

203

204

205

206

207

208

209 significance. $n s: p>0.05 ; * *: p \leq 0.01 ; * * * *: p \leq 0.0001$.
C
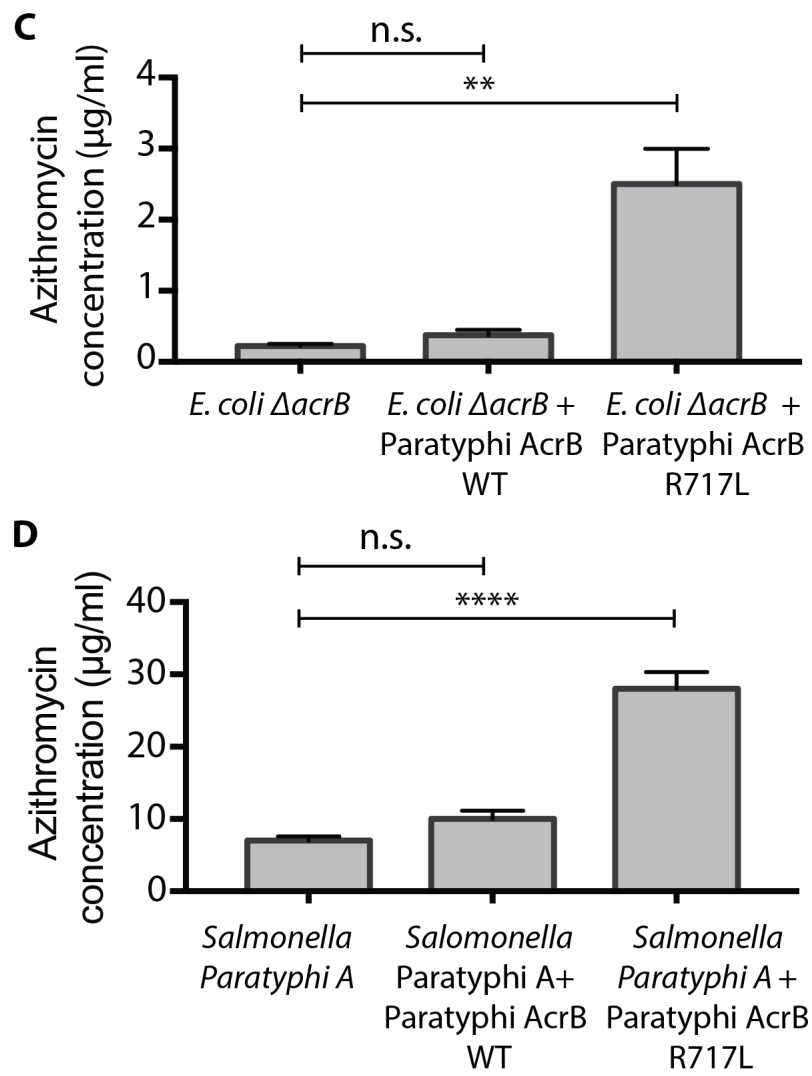

\section{Figure 4: Identification of R717L mutation in AcrB protein in Salmonella Paratyphi A}

\section{strains. (A) Sequence alignment of $a c r B$ gene from the azithromycin-resistant Salmonella}

Paratyphi A strain to the reference strain ATCC 9150. A SNP was identified that changed the R717 to a leucine (L) residue. Quantification of results obtained from three biological replicates for (B) disc diffusion and (C) E-strip assays in E. coli are shown. (D) Quantification of results obtained from at least three biological replicates from azithromycin E-strip assay in Paratyphi strain 4071 is shown. One way-ANOVA with multiple comparisons was used to test statistical 
210 4A). This mutation was absent in the genomes of the Paratyphi A strains in the NCBI database.

211 To determine to effect of R717L mutation, we expressed Paratyphi wild-type AcrB and AcrB-

212 R717L in E. coli $\triangle a c r B$ (Figure 4B, C). As seen for Typhi AcrB R717Q, Paratyphi AcrB-R717L

213 leads to a smaller disc clearance for azithromycin $(26.3 \mathrm{~mm}$ vs $16.3 \mathrm{~mm}, p=0.0001)$ and

214 erythromycin $(22.4 \mathrm{~mm}$ vs $11.4 \mathrm{~mm}, p=0.0001)$ and 10-fold higher azithromycin MIC (0.22

$215 \mu \mathrm{g} / \mathrm{ml}$ vs $2.5 \mu \mathrm{g} / \mathrm{ml}, p=0.003)$. When these plasmids were introduced in a sensitive Paratyphi A

216 strain, we observed 4-fold change in MIC (7 $\mu \mathrm{g} / \mathrm{ml}$ vs $28 \mu \mathrm{g} / \mathrm{ml}, p=0.0001)$ in the presence of

217 the R7171L mutation, confirming that mutations in R717 lead to macrolide resistance.

219 Rising antimicrobial resistance threatens the progress made so far in management of enteric

220 fever. The rate of azithromycin resistance of typhoidal Salmonella in Bangladesh is low and the

221 genetic basis is a chromosomal SNP. However, in light of the outbreak in Pakistan, increasing

222 azithromycin use can place selective pressure on strains such as the ones with R717 mutations to

223 spread. Although no azithromycin resistant XDR isolate has been reported to date, the increasing

224 use of azithromycin and the clear historical record of widespread dissemination of resistance to

225 all previously widely used antimicrobials by Salmonella Typhi and Paratyphi, suggest we will

226 soon face strains resistant to all oral antibiotics. An azithromycin-resistant XDR strain would

227 shift enteric fever treatment from outpatient departments, where patients are currently treated

228 with oral azithromycin, to inpatient departments to be treated with injectable antibiotics like

229 carbapenems, thereby weighing down already struggling health systems in endemic regions

230 (Andrews et al., 2018; Saha et al., 2018a). Moreover, with the dearth of novel antimicrobials in

231 the horizon, we risk losing our primary defense against widespread mortality from enteric fever.

232 In addition to continued surveillance and antimicrobial stewardship, it is imperative to roll-out 
233 the recent WHO prequalified typhoid conjugate vaccine in endemic areas and decrease the

234 overall burden of typhoid.

\section{Materials and Methods}

\section{Study site and population}

238 In this study, we report data from enteric fever surveillance conducted in the inpatient

239 departments of the two largest pediatric hospitals of Bangladesh, Dhaka Shishu (Children)

240 Hospital, DSH, and Shishu Shasthya (Child Health) Foundation Hospital, SSFH. These are

241 sentinel sites of the World Health Organization supported Invasive Bacterial Vaccine

242 Preventable Diseases surveillance platform in Bangladesh.

\section{Patient enrollment, etiology detection and antibiogram}

245 Blood culture was performed at the discretion of the treating physicians. We enrolled patients

246 with positive blood cultures for Salmonella Typhi or Paratyphi A. Blood cultures were

247 performed using standard methods (Saha et al., 2017). We aseptically obtained 2-3 milliliters of

248 blood, which was inoculated into trypticase soy broth supplemented with sodium polyanethole

249 sulphonate $(0.25 \%)$ and isovitalex (1\%). Incubated blood culture bottles were sub-cultured on the

250 second, third, and fifth days of incubation. Identification of Salmonella Typhi/Paratyphi isolates

251 was confirmed using standard biochemical tests and agglutination with Salmonella species and

252 serovar-specific antisera (Ramel, Thermo Fisher Scientific). Laboratory methods for blood

253 culture and organism identification were consistent over the reporting period. 
255 We used disc diffusion methods for determining antibiotic susceptibility patterns for

256 azithromycin, ampicillin, co-trimoxazole, chloramphenicol, ciprofloxacin, levofloxacin,

257 ceftriaxone, cefepime, cefixime and ceftazidime (Oxoid, Thermo Scientific, MA, USA).

258 Azithromycin e-strips (bioMérieux, France) were used to determine the minimum inhibitory

259 concentration (MIC) and confirm azithromycin resistance for strains that exhibited zone of

260 clearance $\leq 12 \mathrm{~mm}$ in the presence of azithromycin discs. All results were interpreted according

261 to the latest Clinical and Laboratory Standards Institute guidelines 2018.

263 DNA extraction and whole genome sequencing

264 We conducted whole genome sequencing on all identified azithromycin-resistant strains (12

265 Salmonella Typhi and 1 Paratyphi A). Isolates were grown in MacConkey agar (Oxoid, UK)

266 overnight and DNA was extracted from a suspension of the overnight culture using the QIAamp

267 DNA minikit (Qiagen, Hilden, Germany). Whole genome sequencing was performed on the

268 Illumina HiSeq 4000 platform to generate 150 bp paired-end reads (Novogene Co. Ltd., Beijing,

269 China). We used SPAdes (Bankevich et al., 2012) to assemble the short paired-end reads into

270 contigs for downstream analyses. All the sequences have been submitted to EnteroBase and

271 NCBI (BioProject ID: PRJNA528114).

272

273 Bioinformatics analysis

274 For comparative genomic analysis, we compared the 12 azithromycin-resistant Salmonella Typhi

275 strains with 462 strains that were previously isolated and genetically characterized by our group

276 in Bangladesh (Tanmoy et al., 2018). Using the ParSNP tool (Treangen et al., 2014), we

277 constructed whole-genome SNP tree using all 475 genomes and determined the genotypes using 
278 srst2 package (Inouye et al., 2014). Ggtree was used to make the phylogenetic tree and overlay

279 the genotype data (Yu et al., 2017). Srst2, ResFinder (Zankari et al., 2012) and CARD (Jia et al.,

280 2017) were used to predict antimicrobial resistance markers, and PlasmidFinder (Carattoli et al.,

$2812014)$ to identify the putative plasmids present in these strains. Finally, we compared the

282 resistant strains to all sensitive Salmonella Typhi strains manually to find SNPs exclusive to the

283 resistant strains (comparison to the most closely related 5 genomes are shown in Figure 1C)

284 using the GinGR tool from the Harvest suite (Treangen et al., 2014). To predict the function of

285 the SNPs on protein function, we examined the protein sequence and conducted structural

286 analyses (Figure 2).

287

288 Macrolide susceptibility test in $E$. coli and Salmonella Typhi

289 We amplified the $a c r B$ genes from azithromycin-resistant Salmonella Typhi strain 5003 and

290 Paratyphi A strain 3144 and azithromycin-sensitive Typhi strain 4119 and Paratyphi A strain

2914071 for downstream cloning. The genes were inserted into the multiple cloning site of

292 pHERD30T using Gibson assembly (Gibson et al., 2009). We verified the sequences of all

293 inserted genes through Sanger sequencing. The plasmids were chemically transformed into

$294 \mathrm{CaCl}_{2}$-competent E. coli BW25112 $\triangle a c r B$ strains, and electroporated into Typhi strain 4119 and

295 Paratyphi A strain 4071. E. coli strains with plasmids (with or without an insert) were tested for

296 susceptibility patterns for erythromycin, azithromycin, ampicillin, co-trimoxazole,

297 chloramphenicol, ciprofloxacin, levofloxacin, ceftriaxone, cefepime, cefixime and ceftazidime

298 using the disc diffusion method, and MIC was determined using azithromycin E-strips. Typhi

299 and Paratyphi strains with plasmids (with or without an insert) were tested for susceptibility

300 patterns for azithromycin using E-strips. 


\section{Acknowledgements}

302 We are thankful to Dr. Shweta Karambelkar, Dr. Balint Csorgo, and Beatriz A. Osuna of

303 University of California, San Francisco, and Sunita Patil of Stanford University for technical

304 assistance with laboratory work and to Arif M. Tanmoy of the Child Health Research Foundation

305 for bioinformation guidance.

\section{Funding}

308 No external funding was attained for this study. The enteric fever surveillance in Dhaka Shishu

309 (Children) Hospital was supported by Gavi, the Vaccine Alliance, through the World health

310 Organization-supported Invasive Bacterial Vaccine Preventable Diseases study (grant numbers

$311201588766,201233523,201022732,200749550,201686542)$

312

\section{Conflict of interest}

314 Authors declare no conflict of interest pertaining to the work presented here. 


\section{References}

315

Ababou A, Koronakis V. 2016. Structures of Gate Loop Variants of the AcrB Drug Efflux Pump Bound by Erythromycin Substrate. PLOS ONE 11:e159154. doi:10.1371/journal.pone.0159154

Andrews JR, Qamar FN, Charles RS, Ryan ET. 2018. Extensively Drug-Resistant Typhoid Are Conjugate Vaccines Arriving Just in Time? NEJM 379:1493-1495.

Balasegaram S, Potter AL, Grynszpan D, Barlow S, Behrens RH, Lighton L, Booth L, Inamdar L, Neal K, Nye K, Lawrence J, Jones J, Gray I, Tolley D, Lane C, Adak B, Cummins A, Addiman S. 2012. Guidelines for the public health management of typhoid and paratyphoid in England: Practice guidelines from the National Typhoid and Paratyphoid Reference Group. Journal of Infection 65:197-213. doi:10.1016/j.jinf.2012.05.005

Bankevich A, Nurk S, Antipov D, Gurevich AA, Dvorkin M, Kulikov AS, Lesin VM, Nikolenko SI, Pham S, Prjibelski AD, Pyshkin AV, Sirotkin AV, Vyahhi N, Tesler G, Alekseyev MA, Pevzner PA. 2012. SPAdes: a new genome assembly algorithm and its applications to single-cell sequencing. J Comput Biol 19:455-477. doi:10.1089/cmb.2012.0021

Blair JMA, Bavro VN, Ricci V, Modi N, Cacciotto P, Kleinekathöfer U, Ruggerone P, Vargiu AV, Baylay AJ, Smith HE, Brandon Y, Galloway D, Piddock LJV. 2015. AcrB drugbinding pocket substitution confers clinically relevant resistance and altered substrate specificity. PNAS 112:3511-3516. doi:10.1073/pnas.1419939112

Carattoli A, Zankari E, García-Fernández A, Larsen MV, Lund O, Villa L, Aarestrup FM, Hasman H. 2014. In Silico Detection and Typing of Plasmids using PlasmidFinder and Plasmid Multilocus Sequence Typing. Antimicrobial Agents and Chemotherapy 58:38953903. doi:10.1128/AAC.02412-14

Chau TT, Campbell JI, Galindo CM, Van Minh Hoang N, Diep TS, Nga TTT, Van Vinh Chau N, Tuan PQ, Page AL, Ochiai RL, Schultsz C, Wain J, Bhutta ZA, Parry CM, Bhattacharya SK, Dutta S, Agtini M, Dong B, Honghui Y, Anh DD, Canh DG, Naheed A, Albert MJ, Phetsouvanh R, Newton PN, Basnyat B, Arjyal A, La TTP, Rang NN, Phuong LT, Van Be Bay P, von Seidlein L, Dougan G, Clemens JD, Vinh H, Hien TT, Chinh NT, Acosta CJ, Farrar J, Dolecek C. 2007. Antimicrobial Drug Resistance of Salmonella enterica Serovar Typhi in Asia and Molecular Mechanism of Reduced 
Susceptibility to the Fluoroquinolones. Antimicrob Agents Chemother 51:4315-4323. doi:10.1128/AAC.00294-07

Dimitrov T, Udo EE, Albaksami O, Kilani AA, Shehab E-DMR. 2007. Ciprofloxacin treatment failure in a case of typhoid fever caused by Salmonella enterica serotype Paratyphi A with reduced susceptibility to ciprofloxacin. Journal of Medical Microbiology 56:277279. doi:10.1099/jmm.0.46773-0

Djeghout B, Saha S, Sajib MSI, Tanmoy AM, Islam M, Kay GL, Langridge GC, Endtz HP, Wain J, Saha SK. 2018. Ceftriaxone-resistant Salmonella Typhi carries an IncI1-ST31 plasmid encoding CTX-M-15. Journal of Medical Microbiology 67:620-627. doi:10.1099/jmm.0.000727

Gibson DG, Young L, Chuang R-Y, Venter JC, Hutchison Iii CA, Smith HO. 2009. Enzymatic assembly of DNA molecules up to several hundred kilobases. Nature Methods 6:343345. doi: $10.1038 /$ nmeth. 1318

Holt KE, Phan MD, Baker S, Duy PT, Nga TVT, Nair S, Turner AK, Walsh C, Fanning S, Emergence of a Globally Dominant IncHI1 Plasmid Type Associated with Multiple Drug Resistant Typhoid. PLoS Negl Trop Dis 5. doi:10.1371/journal.pntd.0001245

Inouye M, Dashnow H, Raven L-A, Schultz MB, Pope BJ, Tomita T, Zobel J, Holt KE. 2014. SRST2: Rapid genomic surveillance for public health and hospital microbiology labs. Genome Medicine 6:90. doi:10.1186/s13073-014-0090-6

Jia B, Raphenya AR, Alcock B, Waglechner N, Guo P, Tsang KK, Lago BA, Dave BM, Pereira S, Sharma AN, Doshi S, Courtot M, Lo R, Williams LE, Frye JG, Elsayegh T, Sardar D, Westman EL, Pawlowski AC, Johnson TA, Brinkman FSL, Wright GD, McArthur AG. 2017. CARD 2017: expansion and model-centric curation of the comprehensive antibiotic resistance database. Nucleic Acids Res 45:D566-D573. doi:10.1093/nar/gkw1004

Kidgell C, Pickard D, Wain J, James K, Diem Nga LT, Diep TS, Levine MM, O’Gaora P, Prentice MB, Parkhill J, Day N, Farrar J, Dougan G. 2002. Characterisation and 
distribution of a cryptic Salmonella typhi plasmid pHCM2. Plasmid 47:159-171. doi:10.1016/S0147-619X(02)00013-6

Klemm EJ, Shakoor S, Page AJ, Qamar FN, Judge K, Saeed DK, Wong VK, Dallman TJ, Nair S, Baker S, Shaheen G, Qureshi S, Yousafzai MT, Saleem MK, Hasan Z, Dougan G, Hasan R. 2018. Emergence of an Extensively Drug-Resistant Salmonella enterica Serovar Typhi

Mirza SH, Beechmg NJ, Hart CA. 1996. Multi-drug resistant typhoid: a global problem. Journal of Medical Microbiology 44:317-319. doi:10.1099/00222615-44-5-317

Molloy A, Nair S, Cooke FJ, Wain J, Farrington M, Lehner PJ, Torok ME. 2010. First Report of Salmonella enterica Serotype Paratyphi A Azithromycin Resistance Leading to Treatment Failure. Journal of Clinical Microbiology 48:4655-4657. doi:10.1128/JCM.00648-10

Nakashima R, Sakurai K, Yamasaki S, Nishino K, Yamaguchi A. 2011. Structures of the multidrug exporter AcrB reveal a proximal multisite drug-binding pocket. Nature 480:565-569. doi:10.1038/nature10641

Park SE, Pham DT, Boinett C, Wong VK, Pak GD, Panzner U, Espinoza LMC, Kalckreuth V von, Im J, Schütt-Gerowitt H, Crump JA, Breiman RF, Adu-Sarkodie Y, Owusu-Dabo E, Rakotozandrindrainy R, Soura AB, Aseffa A, Gasmelseed N, Keddy KH, May J, Sow AG, Aaby P, Biggs HM, Hertz JT, Montgomery JM, Cosmas L, Olack B, Fields B, Sarpong N, Razafindrabe TJL, Raminosoa TM, Kabore LP, Sampo E, Teferi M, Yeshitela B, Tayeb MAE, Sooka A, Meyer CG, Krumkamp R, Dekker DM, Jaeger A, Poppert S, Tall A, Niang A, Bjerregaard-Andersen M, Løfberg SV, Seo HJ, Jeon HJ, Deerin JF, Park J, Konings F, Ali M, Clemens JD, Hughes P, Sendagala JN, Vudriko T, Downing R, Ikumapayi UN, Mackenzie GA, Obaro S, Argimon S, Aanensen DM, Page A, Keane JA, Duchene S, Dyson Z, Holt KE, Dougan G, Marks F, Baker S. 2018. The phylogeography and incidence of multi-drug resistant typhoid fever in sub-Saharan Africa. Nature Communications 9:5094. doi:10.1038/s41467-018-07370-Z

Parry CM, Thieu NTV, Dolecek C, Karkey A, Gupta R, Turner P, Dance D, Maude RR, Ha V, Tran CN, Thi PL, Be BPV, Phi LTT, Ngoc RN, Ghose A, Dongol S, Campbell JI, Thanh DP, Thanh TH, Moore CE, Sona S, Gaind R, Deb M, Anh HV, Van SN, Tinh HT, Day 
NPJ, Dondorp A, Thwaites G, Faiz MA, Phetsouvanh R, Newton P, Basnyat B, Farrar JJ,

Baker S. 2015. Clinically and Microbiologically Derived Azithromycin Susceptibility Agents and Chemotherapy 59:2756-2764. doi:10.1128/AAC.04729-14

Patel SR, Bharti S, Pratap CB, Nath G. 2017. Drug Resistance Pattern in the Recent Isolates of

Pham Thanh D, Karkey A, Dongol S, Ho Thi N, Thompson CN, Rabaa MA, Arjyal A, Holt KE, Basnyat B, Baker S. 2016. A novel ciprofloxacin-resistant subclade of H58 Salmonella Typhi is associated with fluoroquinolone treatment failure. eLife $\mathbf{5}$. doi:10.7554/eLife. 14003

Roumagnac P, Weill F-X, Dolecek C, Baker S, Brisse S, Chinh NT, Le TAH, Acosta CJ, Farrar J, Dougan G, Achtman M. 2006. Evolutionary History of Salmonella Typhi. Science 314:1301-1304. doi:10.1126/science.1134933

Saha Senjuti, Islam M, Saha Shampa, Uddin MJ, Rahman H, Das RC, Hasan M, Amin MR,

Saha Senjuti, Islam M, Uddin MJ, Saha Shampa, Das RC, Baqui AH, Santosham M, Black RE, coordinated Invasive Bacterial-Vaccine Preventable Diseases (IB-VPD) platform: A low cost approach to track an increasingly important disease. PLOS Neglected Tropical Diseases 11:e0005999. doi:10.1371/journal.pntd.0005999

Saha SK, Talukder SY, Islam M, Saha S. 1999. A highly ceftriaxone-resistant Salmonella typhi in Bangladesh. Pediatr Infect Dis J 18:387. 
Sjölund-Karlsson M, Joyce K, Blickenstaff K, Ball T, Haro J, Medalla FM, Fedorka-Cray P, Zhao S, Crump JA, Whichard JM. 2011. Antimicrobial Susceptibility to Azithromycin among Salmonella enterica Isolates from the United States ${ }^{\nabla}$. Antimicrob Agents Chemother 55:3985-3989. doi:10.1128/AAC.00590-11

Tanmoy AM, Westeel E, Bruyne KD, Goris J, Rajoharison A, Sajib MSI, Belkum A van, Saha SK, Komurian-Pradel F, Endtz HP. 2018. Salmonella enterica Serovar Typhi in Bangladesh: Exploration of Genomic Diversity and Antimicrobial Resistance. mBio 9:e02112-18. doi:10.1128/mBio.02112-18

Treangen TJ, Ondov BD, Koren S, Phillippy AM. 2014. The Harvest suite for rapid core-genome alignment and visualization of thousands of intraspecific microbial genomes. Genome Biology 15:524. doi:10.1186/s13059-014-0524-X

Wadsworth CB, Arnold BJ, Sater MRA, Grad YH. 2018. Azithromycin Resistance through Interspecific Acquisition of an Epistasis-Dependent Efflux Pump Component and Transcriptional Regulator in Neisseria gonorrhoeae. mBio 9:e01419-18. doi:10.1128/mBio.01419-18

Wong VK, Baker S, Connor TR, Pickard D, Page AJ, Dave J, Murphy N, Holliman R, Sefton A, Millar M, Dyson ZA, Dougan G, Holt KE, International Typhoid Consortium, Parkhill J, Feasey NA, Kingsley RA, Thomson NR, Keane JA, Weill F-X, Le Hello S, Hawkey J, Edwards DJ, Harris SR, Cain AK, Hadfield J, Hart PJ, Thieu NTV, Klemm EJ, Breiman RF, Watson CH, Edmunds WJ, Kariuki S, Gordon MA, Heyderman RS, Okoro C, Jacobs J, Lunguya O, Msefula C, Chabalgoity JA, Kama M, Jenkins K, Dutta S, Marks F, Campos J, Thompson C, Obaro S, MacLennan CA, Dolecek C, Keddy KH, Smith AM, Parry CM, Karkey A, Dongol S, Basnyat B, Arjyal A, Mulholland EK, Campbell JI, Dufour M, Bandaranayake D, Toleafoa TN, Singh SP, Hatta M, Newton PN, Dance D, Davong V, Onsare RS, Isaia L, Thwaites G, Wijedoru L, Crump JA, De Pinna E, Nair S, Nilles EJ, Thanh DP, Turner P, Soeng S, Valcanis M, Powling J, Dimovski K, Hogg G, Farrar J, Mather AE, Amos B. 2016. An extended genotyping framework for Salmonella 
enterica serovar Typhi, the cause of human typhoid. Nature Communications 7:12827. doi:10.1038/ncomms 12827

Wong VK, Baker S, Pickard DJ, Parkhill J, Page AJ, Feasey NA, Kingsley RA, Thomson NR, Keane JA, Weill F-X, Edwards DJ, Hawkey J, Harris SR, Mather AE, Cain AK, Hadfield J, Hart PJ, Thieu NTV, Klemm EJ, Glinos DA, Breiman RF, Watson CH, Kariuki S, Gordon MA, Heyderman RS, Okoro C, Jacobs J, Lunguya O, Edmunds WJ, Msefula C, Chabalgoity JA, Kama M, Jenkins K, Dutta S, Marks F, Campos J, Thompson C, Obaro S, MacLennan CA, Dolecek C, Keddy KH, Smith AM, Parry CM, Karkey A, Mulholland EK, Campbell JI, Dongol S, Basnyat B, Dufour M, Bandaranayake D, Naseri TT, Singh SP, Hatta M, Newton P, Onsare RS, Isaia L, Dance D, Davong V, Thwaites G, Wijedoru L, Crump JA, De Pinna E, Nair S, Nilles EJ, Thanh DP, Turner P, Soeng S, Valcanis M, Powling J, Dimovski K, Hogg G, Farrar J, Holt KE, Dougan G. 2015. Phylogeographical

Yu EW, Aires JR, McDermott G, Nikaido H. 2005. A Periplasmic Drug-Binding Site of the analysis of the dominant multidrug-resistant H58 clade of Salmonella Typhi identifies inter- and intracontinental transmission events. Nat Genet 47:632-639.

Zankari E, Hasman H, Cosentino S, Vestergaard M, Rasmussen S, Lund O, Aarestrup FM, Chemother 67:2640-2644. doi:10.1093/jac/dks261

$$
\text { Bacteriol 187:6804-6815. doi:10.1128/JB.187.19.6804-6815.2005 }
$$

Yu G, Smith DK, Zhu H, Guan Y, Lam TT-Y. 2017. ggtree: an r package for visualization and annotation of phylogenetic trees with their covariates and other associated data. Methods in Ecology and Evolution 8:28-36. doi:10.1111/2041-210X.12628

$$
\text { Chemother 67:2640-2644. do1:10.1093/jac/dks261 }
$$

\section{Supplementary Figures}




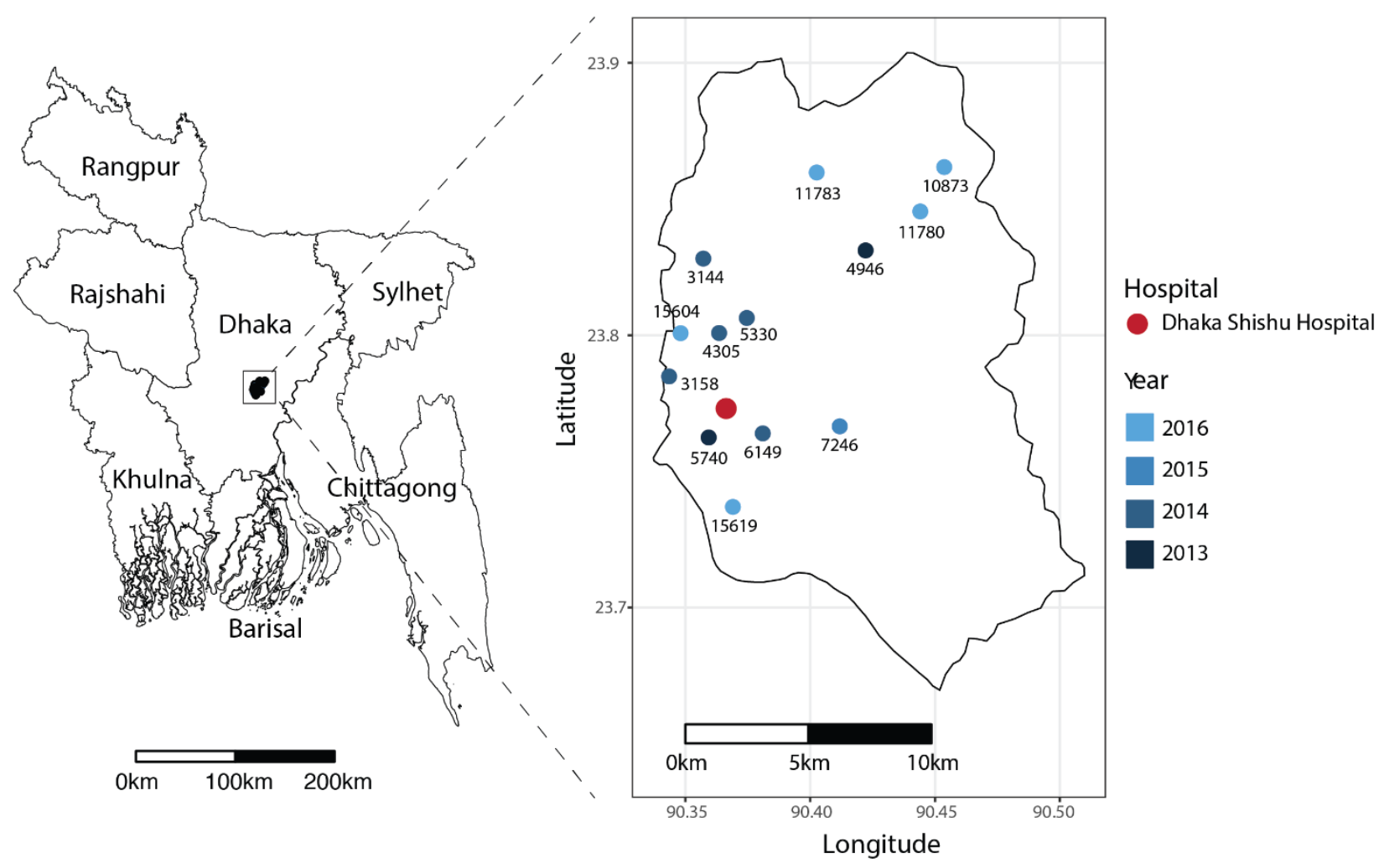

495 Figure S1: Spatiotemporal distribution of azithromycin-resistant Salmonella Typhi and

496 Paratyphi A strains. The 13 azithromycin-resistant typhoidal Salmonella strains were isolated 497 from Dhaka Shishu Hospital (shown in red). All the patients lived within the Dhaka municipal 498 area. 
A

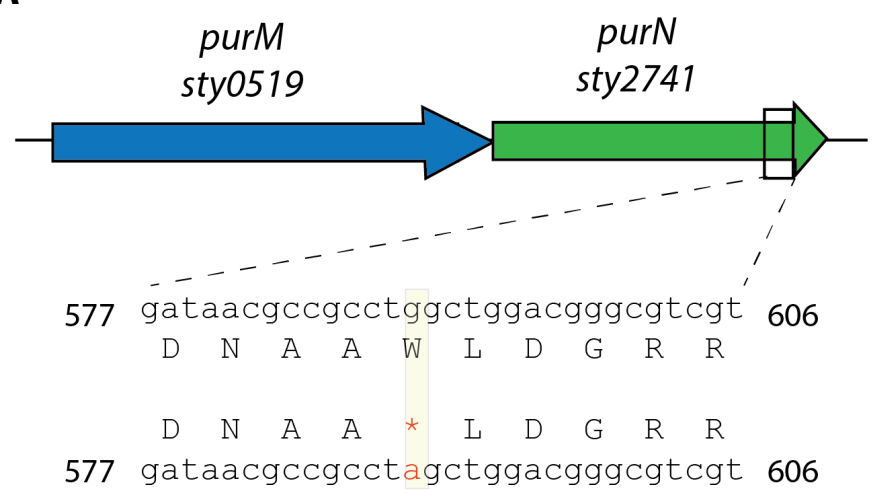

B

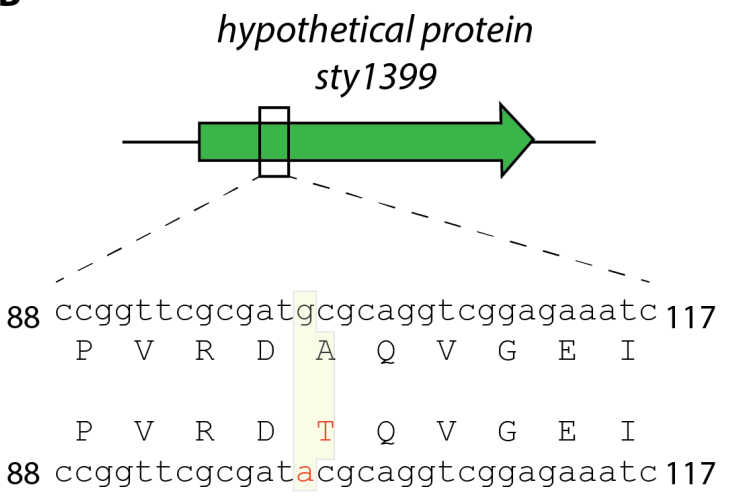

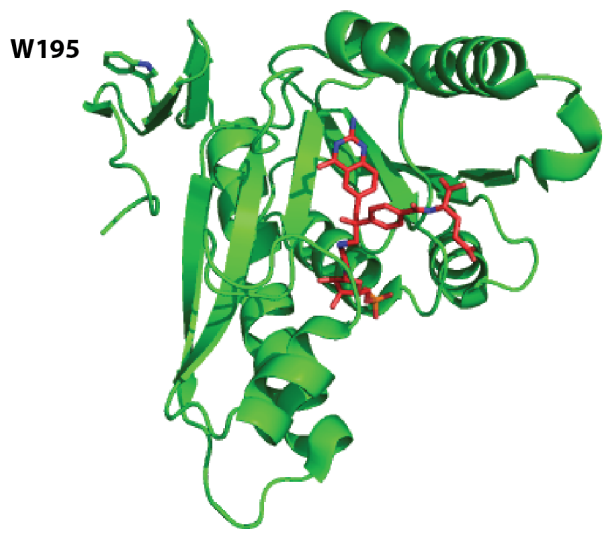

500 Figure S2: Genetic and structural analysis of 2 other non-synonymous SNPs. A) SNP on sty2741 gene (also known as purN) that encodes a glycinamidine ribonucleotide transformyltransferase (GAR-Tfase) enzyme. The SNP leads to change in W195 to a stop codon, leading to premature termination of the protein sequence. The structure of E. coli GAT-Tfase (green, PDB ID: 1C3E) in complex with an inhibitor (shown in red) highlighting the active site is shown. The W195 is present close to the C-terminus and premature termination at this position is predicted to not affect protein function. C) SNP on sty1399 that encodes a hypothetical protein that is proposed to contain a B3/B4 tRNA-binding domain. The function of this protein is not known and the SNP results in conversion of an alanine residue at position 34 to a threonine residue. None of these two genes have been previously implicated in macrolide resistance. 


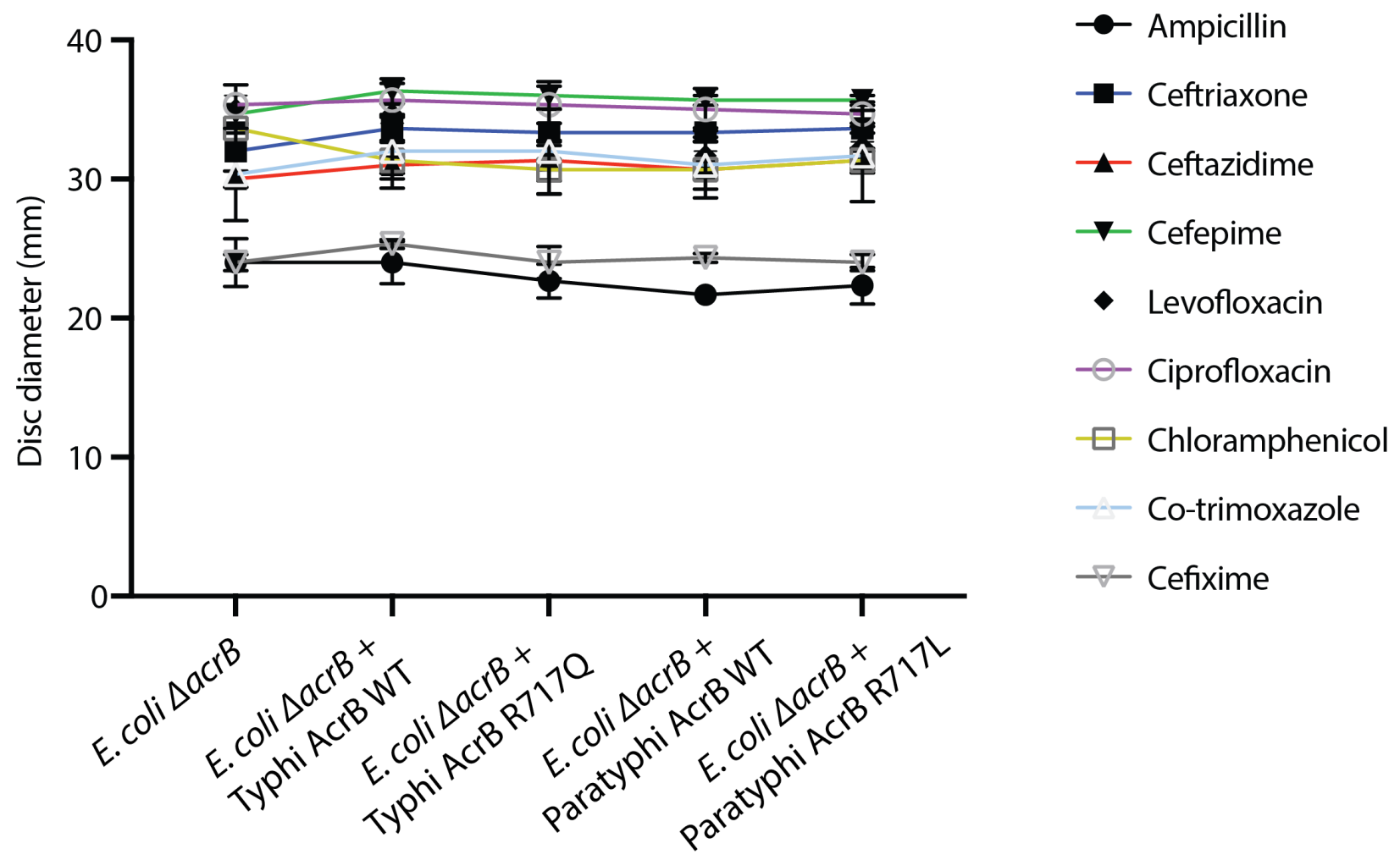

511 Figure S3 - figure supplement 3: AcrB mutations do not affect efflux of other families of

512 antibiotics. Susceptibility of E. coli strains with empty, Salmonella Typhi AcrB WT/R717Q and

513 Paratyphi A AcrB WT/R717L were tested against a panel of 9 different antibiotic including 5

514 beta-lactams, 2 fluoroquinolones, 1 phenicol and 1 diaminopyrimidine /sulphonamide. The data

515 are shown as mean and standard error from 3 different biological replicates. 